\title{
SURVEY ON RELIABLE SLA-BASED MONITORING FOR BILLING SCHEME IN CLOUD COMPUTING
}

\author{
Neha Ramesh Dodke ${ }^{1}$, Sweta Kale ${ }^{2}$ \\ ${ }^{I}$ M.E Student, Dept. of Information Technology, RMD Sinhgad school of Engineering, Maharashtra, India \\ ${ }^{2}$ Asst. Professor, Dept. of Information Technology, RMD Sinhgad school of Engineering, Maharashtra, India
}

\begin{abstract}
The facility to record and keep report of the usage of cloud resources in reliable and certifiable manner is a pioneer to both cloud service provider and to users too. Because usage information is potentially susceptible and must be verifiably correct. This is critical job because in an attempt to provide mutually integrated approach to the system, we come to know that computational overhead increase due to use of traditional asymmetric key operations which lead the system bottleneck. The success of any billing system depends upon factors like integrity, non repudiation. The traditional billing systems are restricted in the security capabilities. To overcome this drawback, paper introduces the billing system called THEMIS. This new billing system introduces some new attributes which provides security facilities to the billing transactions. This system brings new concept called Cloud Notary Authority (CNA) which administer the billing transactions and make it good enough to accept by users and cloud service provider. The Cloud Notary Authority generates the binding information which helps system to solve the future conflicts between users and cloud service provider. SLA (service level agreement) monitoring approach is introduced to provide forgery resistance which doesn't allow to modify the information even by supervisor of the cloud service provider. The service level agreement monitoring approach is improved with TPM (Trusted Platform Module) which sort the information in very secure manner.
\end{abstract}

Keywords - Cloud Notary Authority, Cloud server provider, verification, transaction processing, resource allotment.

\section{INTRODUCTION}

Cloud Computing technology is service oriented technology which define as a type of computing that is based on sharing of resources instead of having confide server, private device to administer the application. In cloud computing, the word cloud refers for the internet and hence this technology also called as Internet based technology. Major cloud providers such as Amazon EC2, S3 [2], Microsoft Azure [3] are becoming popular in current years.

Cloud server provider (CSPs) follows pay-as-you-use mechanism, where users or tenants pay only for the resources that they essentially utilized. This Pay-as-you-use evaluation technique is the chief constitute of cloud environment. CSPs generally assures about the quality of the service in the form of service level agreement (SLA) [4]. SLA is an intermediate between tenants and cloud service provider. It monitors quality of service (QoS) and also defends against forgery and fallacious alteration in data. Although cloud service provider keep track of service billing records but they fails to provide reliability and sureness. It is because tenants or CSPs can modify the records for achieving the trustworthiness even third party can't assure that weather tenant's record is correct or CSPs record is correct. Along with this problem, the capability to record and keep track of usage of cloud resources in acceptable and verifiable way has also become difficult for cloud service provider and users too. Every billing system or transaction must have integrity and no repudiation. The success of such billing system based on these two components. For crystal clear billing, each billing transaction should be prevented against any fraud and false modification. To provide trusted billing system in cloud environment is somewhat tedious for CSPs and tenants [5]. Thus system must be non obstructive and have minimum computation complexity. This paper proposes safe and unthreatened billing scheme called THEMIS. To defeat these drawbacks, THEMIS devised three mechanism namely support for mutually verifiable billing information, trusted SLA monitoring and reduction of computational overhead. The propose system also minimises the expensive public key infrastructure (PKI) [8] computations without weaken the security level of the PKI.

In this paper the billing scheme brings the concept of cloud Notary Authority (CNA) for monitoring the billing transaction. The CNA generates mutually verifiable integrated information which can be used to fix the future controversy between users and CSPs. The system assembles such billing by supervising the service level agreement with the help of S-Mon module. S-Mon has forgery resistive mechanism and it also keeps the track of service logs.

\section{LITERATURE SURVEY}

\subsection{Billing System with Inadequate Security Concerns}

Many researchers studied system like Tivoli [6] for keeping $\operatorname{logs}$ of used resources. But these systems only focuses on resource usage rather than security concerns hence they can't provide integrated transaction. Again these systems designed only for distributed grid surroundings not for payas-you use approach. 


\subsection{Security Improved Billing System}

Several e-payment schemes such as NetPay [7] have been proposed in related work to provide improved billing system. These schemes help users to perform their payment conveniently. However it can't support security features because the systems basically designed only for transaction integrity rather than other main security features.

\section{THE SYSTEM FRAMWORK}

The system THEMIS [1] contains four main components:

\subsection{Cloud Service Provider (CSP)}

The CSPs follows pay-as-you-use mechanism which means user can consumes as many resources as he need and pay only for resources he consumed.

\subsection{Users}

Users also refer as tenants are the thin clients who use resources, services in cloud environment. Each send Checkin message to CSP to start the session and Check-out message to end the session.

\subsection{Cloud Notary Authority (CNA)}

The CAN provides agreeably valid integrity approach that prevents the malicious behaviour of the user or CSP. Whatever information generated by CAN used to solve the future disputes between users and CSPs. For doing so oneway hash chain technique is used.

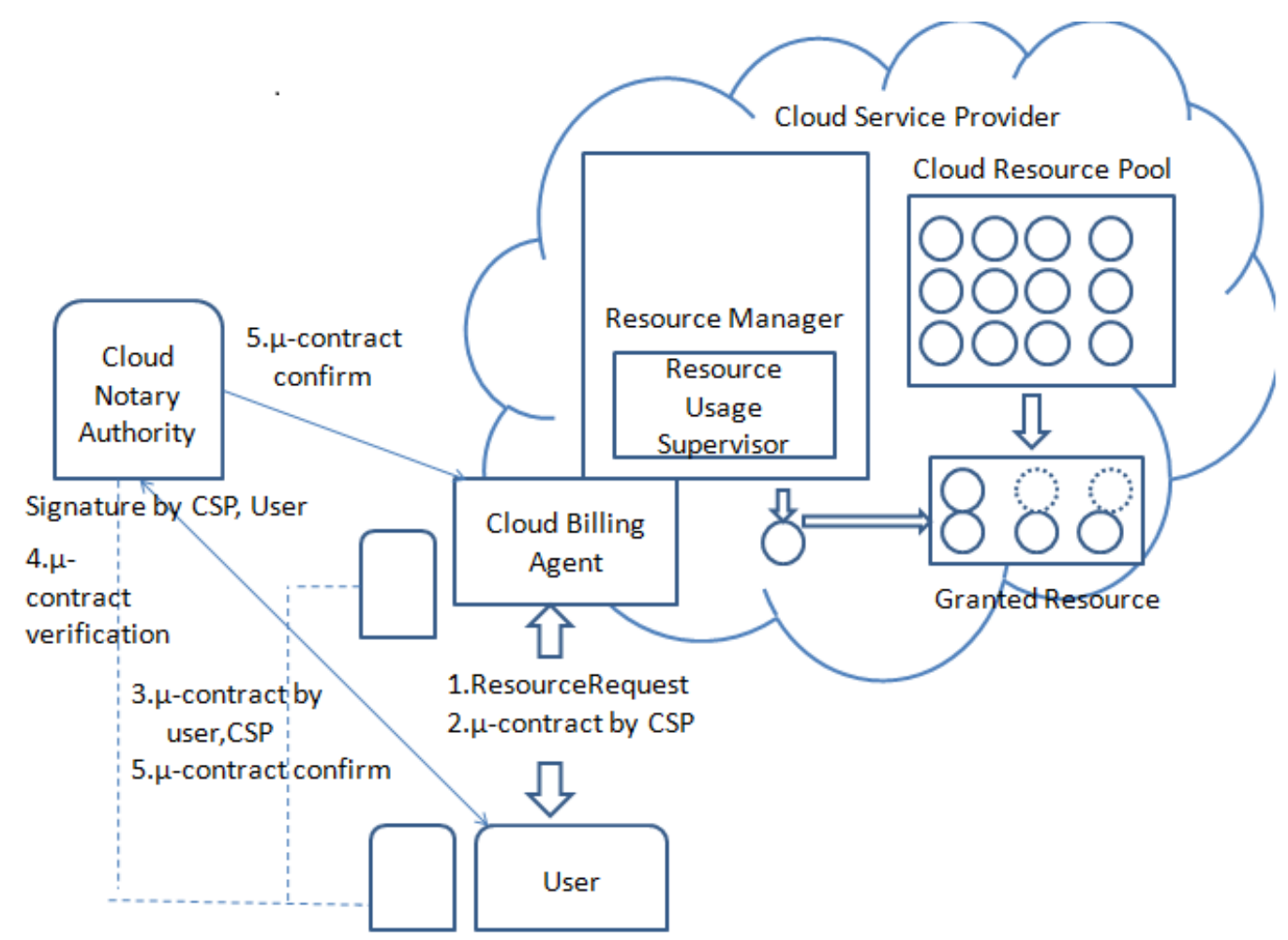

Fig -1: The System Framework

\subsection{S-Mon}

For providing transparent and secure billing transaction in cloud environment service level agreement (SLA) is being monitored by S-Mon. S-Mon offers falsification free SLA monitoring and also keeps track of records of service logs. CNA can get these service logs from S-Mon and stores it in repository for future use. With such system, even administer of CSP can't alter or counterfeit the data.

\section{OVERALL BILLING PROCESS}

In the system architecture, billing transaction can be performed in two kinds of service message namely check-in and check-out. Service check-in message is for initialization of cloud service and service check-out is for termination of the cloud service.

The main steps are as follows:

- User produces service check-in or check-out message and send it to cloud service provider.

- $\quad$ CSP send micro-contract-CSP as a digital signature to user using CSP hash chain.

- $\quad$ By using user's hash chain, users generates digital signature and combine it with CSP's digital signature and send it to CAN.

- $\quad$ CNA authenticate the digital signature from user and CSP and generate integrated binding information

- $\quad$ Once CNA send acknowledgment to user and CSP, the billing process is completed. 
- Lastly according to the service check-in or checkout message, S-Mon sends reports of SLA monitoring to the CNA.
Basically propose billing protocol works in three main steps namely mutually authentication, Hash chain generation and registration, Billing Transaction.

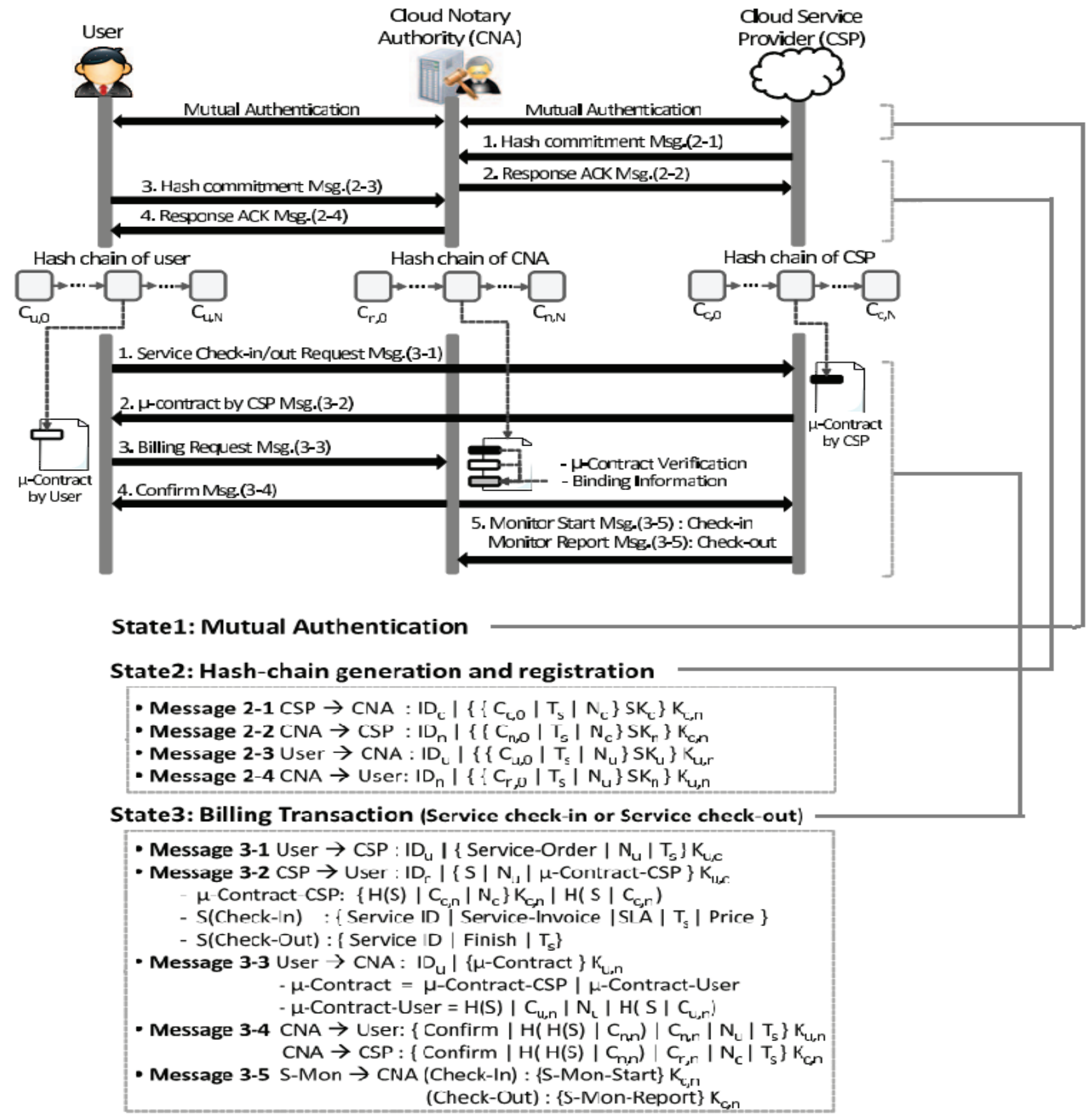

Fig -2: Overall Billing System

As shown in figure 2 the billing protocol works as follows:

- In first state, PKI based operation are performed by the users, CSP and CAN. Throughout mutual verification user, CSP and CNA share the keys.

- The second state is used to generate final hash user and CSP generate the hash chain of length N Using hash function. By digitally signing this final hash they register themselves and key for getting verified by Cloud Notary Authority.

- In this third state, user and CSP generate microcontract message send it to CNA. Once CNA acknowledged them the contract between user and CSP get terminated successfully. 


\section{CONCLUSION}

Our main goal was to provide integrated and secure billing solution for the traditional system. To achieve this goal, we have studied THEMIS billing system which decreases the computational overhead by eliminating the PKI based billing transaction without making any damage to security level of the system. Incorporation with CNA and S-Mon concept billing system easily keep track of logged data and supervise the transactions.

\section{ACKNOWLEDGEMENTS}

I would like to extend my gratitude to many people who helped me to bring this paper fruition. First I would like to thank my guide Prof. Sweta Kale. I am so deeply grateful for her help, professionalism, and valuable guidance throughout this paper. I would also like to thank to my friends and collage. This accomplishment would not have been possible without them.

\section{REFERENCES}

[1]. Ki-Woong Park , Jaesun Han and Kyu Ho Park, "THEMIS: A Mutually Verifiable Billing System for the Cloud Computing Environment," IEEE TRANSACTION ON SERVICES COMPUTING, VOL. 6, NO.3, JULYSEPTEMBER 2013.

[2]. Amazon Web Services, "Amazon Elastic Compute Cloud EC2, Simple Storage Service, "http://aws.amazon.com/ec2, http://aws.amazon.com/s32, Apr. 2011.

[3]. Microsoft, "Microsoft, Windows Azure Platform," http://www.microsoft.com/windowsazure, 2010

[4]. M. Armbrust and A.E. Fox, "Above the Clouds: A Berkeley View of Cloud Computing," Technical Report UCB/EECS-2009-28, Electrical Engineering and Computer Sciences Dept., Univ. of California, Berkeley, Feb. 2009.

[5]. N. Santos, K.P. Gummadi, and R. Rodrigues, "TowardsTrusted Cloud Computing," Proc. Conf. Hot Topics inCloud Computing (HotCloud), 2009.

[6] "Tivoli: Usage and Accounting Manager," IBM press release, 2009.

[7] X. Dai and J. Grundy, "NetPay: An Off-Line, Decentralized Micro-Payment System for Thin-Client Applications," Electronic Commerce Research Applications, vol. 6, pp. 91-101, Jan. 2007.

[8]. G. von Voigt and W. Muller, "Comparison of Grid Accounting Concepts for D-Grid," Proc. Cracow Grid Workshop, pp. 459-466, Oct. 2006

\section{BIOGRAPHIES}

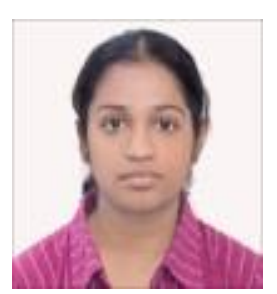

Neha Ramesh Dodke has completed bachelor degree from sant. Gadage baba Amravati University and now M.E Student at RMD Sinhgad school of Engineering, Pune,Maharashtra, India

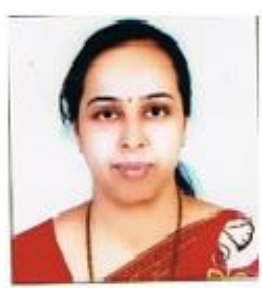

Sweta Kale, Dept. of Information Technology, Working at RMD Sinhgad school of Engineering, Warje Savitribai Phule, Pune university, Maharashtra, India 